IMSc- $92 / 20$

August 20, 2018

\title{
Can Polarised Drell-Yan Shed More Light On The Proton Spin?
}

\author{
Prakash Mathews $^{\dagger}$ and V. Ravindran ${ }^{\ddagger}$ \\ The Institute of Mathematical Sciences, \\ C.I.T. Campus, Madras, 600 113, INDIA.
}

\begin{abstract}
We analyse polarised Drell-Yan process using the factorisation method and derive operator definitions for polarised parton distribution functions. We demonstrate that a factorisation analogous to that in the unpolarised DrellYan case holds in this process. We study the leading order gluonic contribution to the first moment of polarised Drell-Yan function and show that it is consistent with results obtained from polarised deep inelastic scattering.
\end{abstract}

e-mail address:

† prakash@imsc.ernet.in

$\ddagger$ ravi@imsc.ernet.in 
The post Electron Muon Collaboration (EMC) [1] era has seen a revival of interest in the theoretical study of the polarised structure functions of the proton. The EMC experiment (polarised deep inelastic $\mu P$ scattering) gave some surprising results regarding the quark spin contribution to the proton spin, namely, that it is negligibly small. The most appealing interpretation of this result is that not only the quarks but the gluons also can contribute to the proton spin, to the lowest order. This is due to the fact that the product of strong coupling constant, $\alpha_{s}$, and the first moment of polarised gluon distribution function, $\Delta g(x)$, is of order $\alpha_{s}^{0}$ [2, 3]. In the parton model, using the electromagnetic probe to evaluate the gluonic coefficient functions to leading order, one needs a prescription to regulate mass singularities present, and depending on the prescription the gluons may or may not contribute to the first moment of the polarised proton structure function, $g_{1}^{\mu P}(x)$ [3, 4]. Even if one uses the weak probe to evaluate the gluonic coefficient function, one would draw similar conclusions for the moments of the weak structure functions $g_{1,3,4}(x)$ [5]. On the other hand, in the operator product expansion [6] and factorisation method [0, 8], the gluonic coefficient function is free of mass singularities and its first moment vanishes identically giving no gluonic contribution to the first moment of $g_{1}^{\mu P}(x)$ to order $\alpha_{s}$ [8]. Hence, using the factorisation method, in a class of deep inelastic scattering (DIS) processes probed by electromagnetic [8] and weak currents [9], one finds that the gluonic contribution to the first moments of polarised structure functions, $g_{1}^{\mu P}(x)$ and $g_{1,3,4}(x)$, is zero. Under these circumstances, it is therefore instructive to analyse the polarised Drell-Yan (DY) processes as possible sources to measure the gluonic contribution to proton spin. Phenomenologically [10], this process has drawn much attention after the EMC experiment. Further, the experimental possibilities [11 are also bright with the recent technical developments in the acceleration of polarised protons, using the so-called 
Siberian snakes, which facilitate the polarised proton-proton collision at collider energies.

In this letter we analyse the polarised DY process using the factorisation method and show that the polarised DY distribution function $\widetilde{W}\left(\tau, q^{2}\right)$ can be expressed in terms of known operators available in the theory. Further including the appropriate gluonic operators we write a factorisation theorem for polarised DY process and show that the mass singularities factorise leaving the gluonic coefficient functions free of mass singularities to order $\alpha_{s}$.

Consider the longitudinally polarised DY process $P_{1}\left(p_{1}\right)^{\uparrow}+P_{2}\left(p_{2}\right)^{\uparrow \downarrow} \rightarrow \gamma^{*}(q)+X\left(P_{X}\right)$, where $\gamma^{*}$ is the virtual hard photon. The cross section of the process is

$$
\tilde{\sigma}=\frac{1}{2 K(2 \pi)^{3}}\left(-g_{\mu \nu}\right) \int d^{4} q \delta\left(q^{2}-Q^{2}\right) \theta\left(q^{0}\right) \widetilde{W}^{\mu \nu},
$$

where $K$ is the flux factor, $Q^{2}$ is mass of the virtual hard photon, the sum over photon polarisations yields the factor $-g^{\mu \nu}$ and $\widetilde{W}^{\mu \nu}$ is the Fourier transform of product of hadronic currents sandwiched between two polarised proton states. The polarised DY function $\widetilde{W}_{P_{1} P_{2}}\left(\tau, q^{2}\right)$ is defined as

$$
\begin{aligned}
\widetilde{W}_{P_{1} P_{2}}\left(\tau, q^{2}\right)= & -\frac{1}{(2 \pi)^{3}} \int d^{4} q \delta\left(q^{2}-Q^{2}\right) \theta\left(q^{0}\right) \int d^{4} x e^{-i q \cdot x} \\
& <p_{1} s_{1} ; p_{2} s_{2}\left|J_{\mu}(x) \quad J^{\mu}(0)\right| p_{1} s_{1} ; p_{2} s_{2}>_{s p} .
\end{aligned}
$$

We choose the centre of momentum light cone frame where $p_{1}=\left(p_{1}^{+}, 0,0_{\perp}\right), p_{2}=$ $\left(0, p_{2}^{-}, 0_{\perp}\right), q=\left(q^{+}, q^{-}, 0_{\perp}\right), x=\left(x^{+}, x^{-}, x_{\perp}\right)$ and the light cone variables are defined as $p^{ \pm}=\left(p^{0} \pm p^{3}\right) / \sqrt{2}$. Using the DY limit $p_{1}^{+}, p_{2}^{-} \rightarrow \infty, q^{+}, q^{-} \rightarrow \infty$ with $\tau=q^{+} q^{-} / p_{1}^{+} p_{2}^{-}$ ( $\tau=Q^{2} / s$, where $s$ is the cm energy) fixed, we can express the product of hadron currents in terms of the parton currents which are made up of parton field operators coming from two different protons. In order to proceed, we need to express the current-current product so that the dependences on the two proton momenta separate out. We do this by a Fierz 
transformation whereby the product of local bilinear (with respect to two different proton states) operators is expressed as sum of bilocal linear operators. Hence the DY function can be neatly separated out in terms of two proton states and we obtain,

$$
\begin{aligned}
\widetilde{W}_{P_{1} P_{2}}\left(\tau, q^{2}\right)= & \sum_{i} \frac{e_{i}^{2}}{24 \pi} \int \frac{d q^{+}}{q^{+}} \frac{d q^{-}}{q^{-}} \delta\left(1-\frac{Q^{2}}{2 q^{+} q^{-}}\right) \int d x^{+} d x^{-} e^{-i q^{+} x^{-}} e^{-i q^{-} x^{+}} \\
& {\left[<p_{1} s_{1}\left|\bar{\psi}_{a}\left(x^{+}, x^{-}\right) \gamma_{\mu} \gamma_{5} \psi_{a}(0)\right| p_{1} s_{1}>\times\right.} \\
& \left.<p_{2} s_{2}\left|\bar{\psi}_{b}(0) \gamma^{\mu} \gamma_{5} \psi_{b}\left(x^{+}, x^{-}\right)\right| p_{2} s_{2}>+(a \leftrightarrow b)\right]
\end{aligned}
$$

where $i$ runs over different quark flavours, and the scalar, vector and pseudoscalar terms which appear in the Fierz identity do not contribute for the polarised DY process. From the asymptotic behaviour of Fourier transform of singular functions, it is easy to see that the dominant contribution comes from the region near the singularities [12]. Hence, using $q^{+}=x_{a} p_{1}^{+}$and $q^{-}=x_{b} p_{2}^{-}$, we have

$$
\begin{aligned}
\widetilde{W}_{P_{1} P_{2}}\left(\tau, q^{2}\right)= & \sum_{i} \int \frac{d x_{a}}{x_{a}} \frac{d x_{b}}{x_{b}} \frac{2 \pi e_{i}^{2}}{3} \delta\left(1-\frac{\tau}{x_{a} x_{b}}\right) \\
& {\left[\frac{1}{4 \pi} \int d x^{-} e^{-i x_{a} p_{1}^{+} x^{-}}<p_{1} s_{1}\left|\bar{\psi}_{a}\left(x^{-}\right) \gamma^{+} \gamma_{5} \psi_{a}(0)\right| p_{1} s_{1}>\times\right.} \\
& \left.\frac{1}{4 \pi} \int d x^{+} e^{-i x_{b} p_{2}^{-} x^{+}}<p_{2} s_{2}\left|\bar{\psi}_{b}(0) \gamma^{-} \gamma_{5} \psi_{b}\left(x^{+}\right)\right| p_{2} s_{2}>+(a \leftrightarrow b)\right],
\end{aligned}
$$

where we have also used the fact that $<p^{ \pm} s\left|\bar{\psi} \gamma^{\mp} \gamma_{5} \psi\right| p^{ \pm} s>=0$. The above expression provides neat separation of short and long distance physics. By noting that the Dirac delta function corresponds to the $q, \bar{q} \rightarrow \gamma^{*}$ subprocess, the terms in the bracket can be identified with the polarised quark and antiquark distribution functions of the proton, which are consistent with the DIS polarised distribution functions [8] and hence confirms the universality of the parton distribution functions.

To make the definitions of the parton distribution functions gauge invariant, one has to insert the path ordered exponential given below. With this, one can generalise the 
above expression by replacing delta function by hard scattering coefficient function $\widetilde{H}_{a b}$ and adding appropriate gluonic operators of the theory. We thus have

$$
\begin{aligned}
\widetilde{W}_{P_{1} P_{2}}\left(\tau, q^{2}\right)= & \sum_{a, b} \int_{\tau}^{1} \frac{d x_{a}}{x_{a}} \int_{\frac{\tau}{x_{a}}}^{1} \frac{d x_{b}}{x_{b}} f_{\frac{\Delta a}{P_{1}}}\left(x_{a}, \mu^{2}\right) \widetilde{H}_{a b}\left(\frac{\tau}{x_{a} x_{b}}, \frac{q^{2}}{\mu^{2}}\right) f_{\frac{\Delta b}{P_{2}}}\left(x_{b}, \mu^{2}\right)+ \\
& (a \leftrightarrow b)+\cdots,
\end{aligned}
$$

where $\mu^{2}$ is the factorisation scale, $\widetilde{W}_{P_{1} P_{2}}=\frac{1}{2}\left(W_{++}-W_{+-}\right), f_{\Delta a / A}$ is the polarised probability distribution of the parton $a\left(\equiv q_{i}, \bar{q}_{i}, g\right)$ in the polarised hadron target A, defined as $f_{\Delta a / A_{+}}=\left(f_{a_{+} / A_{+}}-f_{a_{-} / A_{+}}\right)$and $\widetilde{H}_{a b}$ is the polarised hard scattering coefficient defined as $\widetilde{H}_{a b}=\frac{1}{2}\left(H_{a_{+} b_{+}}-H_{a_{+} b_{-}}\right)$where the $+(-)$sign denotes the polarisation of the proton or parton along (opposite to) the beam axis. The ellipses in eq.(5) represent the higher twist contributions. The gauge invariant definitions of polarised quark and antiquark distributions in the proton are identified using eq.(田) in terms of the connected matrix elements of the bilocal operators as

$$
\begin{aligned}
& f_{\frac{\Delta q}{A}}(x)=\frac{1}{4 \pi} \int d \xi^{-} e^{-i x \xi^{-} p^{+}}\left[<p, s\left|\bar{\Psi}_{a}\left(0, \xi^{-}, 0_{\perp}\right) \gamma^{+} \gamma_{5} \mathcal{G}_{b}^{a} \Psi^{b}(0)\right| p, s>_{c}\right] \\
& f_{\frac{\Delta \bar{q}}{A}}(x)=\frac{1}{4 \pi} \int d \xi^{-} e^{-i x \xi^{-} p^{+}}\left[<p, s\left|\bar{\Psi}_{a}(0) \gamma^{+} \gamma_{5} \mathcal{G}_{b}^{a \dagger} \Psi^{b}\left(0, \xi^{-}, 0_{\perp}\right)\right| p, s>_{c}\right] .
\end{aligned}
$$

In terms of the appropriate gluonic operators in the theory the gluonic distribution function is defined as

$$
\begin{aligned}
f_{\frac{\Delta g}{A}}(x)= & \frac{i}{4 \pi x p^{+}} \int d \xi^{-} e^{-i x \xi^{-} p^{+}}\left[<p, s\left|F_{a}^{+\mu}\left(0, \xi^{-}, 0_{\perp}\right) \mathcal{G}_{b}^{a} \quad \widetilde{F}_{\mu}^{+b}(0)\right| p, s>_{c}-\right. \\
& \left.<p, s\left|F_{a}^{+\mu}(0) \mathcal{G}_{b}^{a} \widetilde{F}_{\mu}^{+b}\left(0, \xi^{-}, 0_{\perp}\right)\right| p, s>_{c}\right]
\end{aligned}
$$

where $F_{a}^{\mu \nu}$ is the gluon field strength operator, $\mathcal{G}^{a}{ }_{b}=\mathcal{P} \exp \left[i g \int_{0}^{\xi^{-}} d \zeta^{-} A^{+}\left(0, \zeta^{-}, 0_{\perp}\right)\right]_{b}^{a}, \mathcal{P}$ denoting the path ordering of the gluon field operators $A^{\mu}$. For known targets like quarks 
and gluons the distribution functions are normalised as

$$
\begin{aligned}
f_{\frac{\Delta q+\Delta \bar{q}}{a(h)}}(z) & =h \delta(1-z) \delta_{a,(q, \bar{q})} \\
f_{\frac{\Delta g}{a(h)}}(z) & =h \delta(1-z) \delta_{a, g}
\end{aligned}
$$

where $h= \pm 1$ denotes the helicity of the incoming parton. In general, the above matrix elements cannot be calculated within the domain of perturbative QCD, owing to the complex nature of the proton target. But these are calculable for specific parton targets like quarks and gluons. Leading order corrections to these distributions are both ultraviolet (UV) and mass sensitive. These can be regularised and renormalised in any scheme. We use dimensional regularisation to regularise the UV divergences and $\overline{M S}$ scheme to renormalise these distributions. This choice is preferred as it ensures gauge invariance and relativistic invariance although it destroys scale invariance. The mass singularities are avoided by considering on-shell quarks in the cut quark loop and off-shell gluons. Unlike the distribution functions $f_{\Delta a / A}$, the hard scattering coefficients are determined in the theory without any reference to the target. These coefficients may also be mass and UV sensitive and should be regularised in the same scheme.

The $n^{\text {th }}$ moment of the DY function can be related to the $n^{\text {th }}$ moment of the distribution functions and the hard scattering coefficients as

$$
\widetilde{W}_{A B}\left(n, q^{2}\right)=\sum_{a b} f_{\frac{\Delta a}{A}}\left(n, \mu^{2}\right) \widetilde{H}_{a b}\left(n, \frac{q^{2}}{\mu^{2}}\right) f_{\frac{\Delta b}{B}}\left(n, \mu^{2}\right),
$$

where $f(n)=\int_{0}^{1} x^{n-1} f(x) d x$. Here we can easily identify $f_{\Delta a / A}\left(n, \mu^{2}\right)$ with the matrix elements of the twist two local operators.

One of the interesting offshoots of the EMC interpretation is that $\alpha_{s} \Delta g$ is of order $\alpha_{s}^{0}$. Hence the leading order contribution to the DY process comes from the following subprocesses $q \bar{q} \rightarrow \gamma^{*}, g(q, \bar{q}) \rightarrow \gamma^{*}(q, \bar{q})$ and $g g \rightarrow \gamma^{*} q \bar{q}$. We therefore use the factorisation 
theorem to calculate the gluonic contribution to the spin dependent DY function to order $\alpha_{s}$ and the quark contribution to lowest order. To this order, the quantity of interest is

$$
\begin{aligned}
\widetilde{W}_{P_{1} P_{2}}\left(\tau, q^{2}\right)= & \sum_{i} \int_{\tau}^{1} \frac{d x_{a}}{x_{a}} \int_{\frac{\tau}{x_{a}}}^{1} \frac{d x_{b}}{x_{b}} f_{\Delta q_{i} / P_{1}}\left(x_{a}, \mu^{2}\right) \widetilde{H}_{q_{i} \bar{q}_{i}}\left(\frac{\tau}{x_{a} x_{b}}, \frac{q^{2}}{\mu^{2}}\right) f_{\Delta \bar{q}_{i} / P_{2}}\left(x_{b}, \mu^{2}\right)+ \\
& \sum_{i} \int_{\tau}^{1} \frac{d x_{a}}{x_{a}} \int_{\frac{\tau}{x_{a}}}^{1} \frac{d x_{b}}{x_{b}} f_{\Delta q_{i} / P_{1}}\left(x_{a}, \mu^{2}\right) \widetilde{H}_{q_{i} g}\left(\frac{\tau}{x_{a} x_{b}}, \frac{q^{2}}{\mu^{2}}\right) f_{\Delta g / P_{2}}\left(x_{b}, \mu^{2}\right)+ \\
& \sum_{i} \int_{\tau}^{1} \frac{d x_{a}}{x_{a}} \int_{\frac{\tau}{x_{a}}}^{1} \frac{d x_{b}}{x_{b}} f_{\Delta \bar{q}_{i} / P_{1}}\left(x_{a}, \mu^{2}\right) \widetilde{H}_{\bar{q}_{i} g}\left(\frac{\tau}{x_{a} x_{b}}, \frac{q^{2}}{\mu^{2}}\right) f_{\Delta g / P_{2}}\left(x_{b}, \mu^{2}\right)+ \\
& (a \leftrightarrow b) .
\end{aligned}
$$

Using the Born diagram (fig.1), we get

$$
\widetilde{H}_{q \bar{q}}(\hat{\tau})=\frac{2 \pi e_{i}^{2}}{3} \delta(1-\hat{\tau})
$$

To evaluate the gluonic contribution to the DY function $\widetilde{W}_{P_{1} P_{2}}\left(\tau, q^{2}\right)$ we replace the proton targets by quark and gluons and use eq.(6), eq.(7), eq.(8) and eq.(12) and express the resulting 'compton' subprocess to order $\alpha_{s}$ as

$$
\begin{aligned}
\widetilde{W}_{q g}\left(\hat{\tau}, q^{2}\right)= & \sum_{i} \int_{\hat{\tau}}^{1} \frac{d y_{a}}{y_{a}} \int_{\frac{\hat{\gamma}}{y_{a}}}^{1} \frac{d y_{b}}{y_{b}} f_{\Delta \bar{q}_{i} / g}\left(y_{a}, \mu^{2}\right) \widetilde{H}_{q_{i} \bar{q}_{i}}\left(\frac{\hat{\tau}}{y_{a} y_{b}}, \frac{q^{2}}{\mu^{2}}\right) f_{\Delta q_{i} / q}\left(y_{b}, \mu^{2}\right)+ \\
& \sum_{i} \int_{\hat{\tau}}^{1} \frac{d y_{a}}{y_{a}} \int_{\frac{\hat{y}}{y_{a}}}^{1} \frac{d y_{b}}{y_{b}} f_{\Delta q_{i} / q}\left(y_{a}, \mu^{2}\right) \widetilde{H}_{q_{i} g}\left(\frac{\hat{\tau}}{y_{a} y_{b}}, \frac{q^{2}}{\mu^{2}}\right) f_{\Delta g / g}\left(y_{b}, \mu^{2}\right),
\end{aligned}
$$

where $m$ is the mass of the quark, $k^{2}$ is off-shell mass of the gluon and $\hat{\tau}\left(\equiv \tau / x_{a} x_{b}\right)$ is the DY variable in the parton level. The only unknown in eq.(14) is $f_{\Delta \bar{q} / g}$ to order $\alpha_{s}$, which is evaluated using eq.(7) in the dimensional regularisation scheme. The only contribution to $f_{\Delta \bar{q} / g(h)}$ comes from the cut triangle diagram (fig. 2) with quark and antiquark loops. The matrix element is only UV divergent if we keep quarks on shell and gluon off-shell. On regulating we get

$$
f_{\frac{\Delta \bar{q}_{i}}{g(h)}}\left(\hat{\tau}, \mu_{R}^{2}\right)=h \frac{\alpha_{s}}{4 \pi}\left[(2 \hat{\tau}-1) \ln \frac{\mu_{R}^{2}}{m^{2}-k^{2} \hat{\tau}(1-\hat{\tau})}-1+\frac{m^{2}}{m^{2}-k^{2} \hat{\tau}(1-\hat{\tau})}\right]
$$


where $\mu_{R}^{2}=\mu^{2} \exp \left(\gamma_{E}+\frac{2}{\epsilon}\right)$ and $h$ is the helicity of the gluon target. Since $\widetilde{H}_{q \bar{q}}$ is given in eq. (13), $\widetilde{H}_{q g}$ can be found if $\widetilde{W}_{q g}$ is calculated. As the compton subprocess diagram (fig.3) has only mass singularities, we keep all the masses non zero. Neglecting the power corrections in the large $q^{2}$ and $s$ limit, we get

$$
\begin{aligned}
\widetilde{W}_{q g}\left(\hat{\tau}, q^{2}\right)= & \sum_{i} \alpha_{s} \frac{e_{i}^{2}}{6}\left[(2 \hat{\tau}-1) \ln \frac{q^{2}(1-\hat{\tau})^{2}}{\hat{\tau}\left(m^{2}-k^{2} \hat{\tau}(1-\hat{\tau})\right)}-\frac{1}{2}\left(3 \hat{\tau}^{2}+2 \hat{\tau}-3\right)+\right. \\
& \left.\frac{m^{2}}{m^{2}-k^{2} \hat{\tau}(1-\hat{\tau})}\right] .
\end{aligned}
$$

Now we can evaluate the hard scattering coefficient, by using the above results

$$
\begin{aligned}
\widetilde{H}_{q g}\left(\hat{\tau}, q^{2}\right) & =-\sum_{i} \alpha_{s} \frac{e_{i}^{2}}{6}\left[(2 \hat{\tau}-1)\left(\ln \frac{\hat{\tau}}{(1-\hat{\tau})^{2}}+\ln \frac{\mu_{R}^{2}}{q^{2}}\right)+\frac{1}{2}\left(3 \hat{\tau}^{2}+2 \hat{\tau}-3\right)-1\right] \\
& =\widetilde{H}_{\bar{q} g}\left(\hat{\tau}, q^{2}\right)
\end{aligned}
$$

We find that a similar analysis of the weak DY processes also yields the same quark and gluon hard scattering coefficient functions but for weak coupling factors. Note that the above expression is free of any mass singularities, verifying the proposed factorisation to order $\alpha_{s}$ in the gluonic sector. In addition, since the first moment of $\widetilde{H}_{q g}\left(\hat{\tau}, q^{2}\right)$ vanishes, there is no gluonic contribution to the first moment of DY function through the 'compton' subprocess for both electromagnetic and weak processes.

In the parton model analysis of the above problem, the calculation of gluonic coefficient function in the DY function involves only $\widetilde{W}_{q g}\left(\hat{\tau}, q^{2}\right)$ term. This being mass singular, a particular choice of prescription has to be made to regulate these singularities. Depending on the prescription one gets different results: for $m^{2} \neq 0, k^{2}=0$ the first moment of $\widetilde{W}_{q g}\left(\hat{\tau}, q^{2}\right)$ is zero, while for $m^{2}=0, k^{2} \neq 0$ we have,

$$
\int d \hat{\tau} \widetilde{W}_{q g}\left(\hat{\tau}, q^{2}\right)=-\frac{1}{2}\left(\frac{\alpha_{s} e_{i}^{2}}{3}\right) .
$$


It is interesting to note that one arrives at the same conclusion even in the polarised DIS case. Invoking universality of parton distributions we expect the first moment of $\widetilde{W}_{P_{1} P_{2}}\left(\tau, q^{2}\right)$ in the parton model using the prescription $m^{2}=0, k^{2} \neq 0$, to be of the form $\Delta q^{\prime} \Delta \bar{q}^{\prime}$, where $\Delta q^{\prime}=\Delta q-\left(\alpha_{s} / 4 \pi\right) \Delta g$ [3]. Note that eq.(18) verifies this to order $\alpha_{s}$. This implies that the first moment of $\widetilde{W}_{g g}\left(\hat{\tau}, q^{2}\right)$ is $\left(4 \pi e_{i}^{2} / 3\right)\left(\alpha_{s} / 4 \pi\right)^{2}$. Assuming this to be true in the proposed factorisation scheme, one finds that the first moment of $\widetilde{H}_{g g}\left(\hat{\tau}, q^{2}\right)$ is also zero. Explicit computation of $\widetilde{H}_{g g}$ involves evaluation of eight diagrams and three body phase space integral. We will be presenting these details elsewhere and expect that it will corroborate that the first moment vanishes. Hence, in the factorisation scheme, gluons can not contribute to the first moment of polarised DY function.

We have studied the polarised DY process using in the factorisation method and provide operator definitions for the polarised parton distribution functions, which are consistent with that of DIS, hence confirms universality. We have explicitly shown the factorisation of mass singularities to order $\alpha_{s}$ in the gluonic sector. The gluonic contribution to the first moment of the polarised DY function from the 'compton' subprocess turns out to be zero. Invoking universality we conclude that the gluonic contribution to the first moment of polarised DY function vanishes.

We are grateful to G. Date, D. Indumathi, M.V.N. Murthy, R. Ramachandran and S. Umasankar for useful discussions and suggestions. Further we thank DI, MVNM, RR and SU for carefully reading the manuscript and making useful suggestions. 


\section{References}

[1] J. Ashman et al. , Phys. Lett. B(1989).; J. Ashman et al. Nucl.Phys. B328 (1989) 1.

[2] C.S. Lam and Bing-An Li, Phys. Rev. D25 (1982) 683;

[3] G. Altarelli and G.G. Ross, Phys. Lett B212 (1988) 391.

[4] A.V. Efremov and O.V. Teryaev, In Proceedings of the International Hadron Symposium, Bechyne, Czechoslovakia, 1988, ed. Fischer etal. (Czechoslovakian Academy of Science, Prague, 1989), p. 302; R.D. Carlitz, J.C. Collins and A.H. Mueller, Phys. Lett. B214 (1988) 229.

[5] Prakash Mathews and V. Ravindran, Phys. Lett. B 278 (1992) 175; W. Vogelsang and A. Weber, Nucl. Phys B362(1991) 3.

[6] J. Kodaira et.al. Nucl. Phys. B165(1980) 129; K. Hagiwara, Suppl. Prog. Theor. Phys. 77(1983) 100; T. Muta, Foundations of Quantum Chromodynamics (World Scientific 1987).

[7] J.C. Collins, D.E. Soper and G. Sterman, Advanced series on Directions in High Energy Physics -Vol 5 (Perturbative QCD) ed. A.H. Mueller; G. Sterman, Lecture Notes, ITP-SB-91-55.

[8] G.T. Bodwin and J. Qiu Phys Rev D41 (1990) 2755; A.V. Manohar Phys. Rev. Lett. 65(1990) 2511; A.V. Manohar, Phys. Rev. Lett, 66(1991) 289.

[9] Prakash Mathews and V. Ravindran, imsc 91/32 (1991) to appear in IJMPA. 
[10] D. Indumathi, S. Gupta and M.V.N. Murthy, Z. Phys. C42 (1989) 493; ibid C47 (1990) 227; H.Y. Cheng, S.N. Lai, Phys. Rev. D41 (1990) 91; Prakash Mathews, R. Ramachandran, Z. Phys. 53 (1992) 305.

[11] G. Bunce et.al. Particle World 3 (1992) 1.

[12] M.J. Lighthill, Introduction to Fourier Analysis and Generalised Functions (Cambridge University Press, Cambridge, 1958); R.L Jaffe, Relativistic Dynamics and Quark-Nuclear Physics (John Wiley and sons 1986) ed. M.B. Johnson, A. Picklesimer. 


\section{Figure Captions}

Fig 1. Quark Born diagram.

Fig 2. Triangle diagrams contributing to polarised distribution functions $f_{\Delta q / g(h)}$.

Fig 3. 'Compton' subprocess. 